\title{
Editorial
}

Nephrology

Published online: June 28, 2016

\section{Copeptin and Decline in Kidney Function}

\author{
Jingyin Yan ${ }^{a}$ Sankar D. Navaneethan ${ }^{a, b}$ \\ a Department of Medicine, Selzman Institute for Kidney Health, Section of Nephrology, Baylor College of Medicine,



Arginine vasopressin (AVP) is an important pituitary hormone in humans that mediates osmoregulation, water balance and tonicity. Disturbances in AVP regulation contribute to the pathogenesis of diabetes insipidus and cardiovascular diseases [1]. However, the measurement of endogenous plasma AVP level is difficult due to technical limitations and not widely available. Copeptin, a 39 glycosylated amino acid, is the carboxyl-terminal part of precursor-AVP and is released from the hypothalamus synergistically with AVP. It is easier to measure copeptin rather than AVP in serum due to its superior stability. Previous studies have demonstrated high functional correlation between copeptin and mature AVP supporting it as an appropriate surrogate marker in lieu of AVP [1]. Copeptin is partly cleared by the kidneys and hence, the level of copeptin is higher in chronic kidney disease (CKD) or end-stage renal disease (ESRD) patients, compared to those with preserved kidney function [2].

Associations between copeptin and development of heart failure, myocardial infarction, diabetes insipidus, hyponatremia, diabetes mellitus (DM) and metabolic syndrome have been reported. The importance of copeptin in kidney diseases has also been illustrated in the recent literature. In those with ESRD and type 2 DM, higher copeptin level was associated with an increased risk of stroke, sudden cardiac death and all-cause mortal- ity [3]. An elevated copeptin level was noted to be associated with reduced estimated glomerular filtration rate (eGFR) and simple renal cyst growth in patients with autosomal dominant polycystic kidney disease (ADPKD) [1]. Therefore, it is proposed that copeptin can serve as a marker of the progression of renal cystic disease. In renal transplant recipients, an association between baseline copeptin and deterioration of allograft function has been reported [2]. In type 2 diabetic population, high copeptin level at baseline was associated with decline in kidney function during follow-up. However, whether copeptin levels could help us predict incident CKD in general population has not been well-studied.

In this issue of the Journal, Tasevska et al. [4] examined the associations between plasma copeptin and incident CKD (defined as eGFR $<60 \mathrm{ml} / \mathrm{min} / 1.73 \mathrm{~m}^{2}$ ) in a Northern European population. This is a secondary analysis of Malmö Diet and Cancer Study (MDCS), a large prospective population-based cohort in Sweden designed to study the association between Western diet, life style and cancer risks. About 28,000 middle-aged individuals were enrolled in MDCS with baseline examination conducted between 1991 and 1996. Among them, approximately 6,000 subjects were randomly selected to participate in the MDCS Cardiovascular Cohort study. About half of the individuals in the MDCS Cardiovascular Cohort study

\section{KARGER}

E-Mail karger@karger.com

www.karger.com/ajn
(C) 2016 S. Karger AG, Basel

0250-8095/16/0441-0019\$39.50/0
Sankar D. Navaneethan, MD, MS, MPH

Section of Nephrology, Baylor College of Medicine

One Baylor Plaza, Suite 100-37D

Houston, TX 77030 (USA)

E-Mail sankar.navaneethan @bcm.edu 
were eligible and included in this analysis. Plasma copeptin level was measured in the samples obtained at the baseline visit of the study using a commercially available assay and was categorized into quintiles. At baseline, eGFR was $74.7 \pm 14.6 \mathrm{ml} / \mathrm{min} / 1.73 \mathrm{~m}^{2}$ using the Modification of Diet in Renal Disease Formula equation. Patients were followed for $16.6 \pm 1.5$ years. In the multivariable analysis, after adjusting for relevant covariates including eGFR, each standard deviation increase in copeptin levels was associated with greater decline in kidney function and eGFR $<60 \mathrm{ml} / \mathrm{min} / 1.73 \mathrm{~m}^{2}$ (OR 1.19, 95\% CI 1.04-1.36; $\mathrm{p}=0.01$ ). Moreover, the strength of these associations increased linearly with higher risk estimates for those with higher levels of copeptin. They noted qualitatively similar results in analyses using the CKDEPI equation.

A large sample size and longer follow-up are the major strengths of this prospective cohort study. Also, the findings are consistent with another prospective cohort study in a French community, which showed similar associations between the baseline copeptin levels and development of CKD over a 9-year period [5]. However, some key limitations are worth noting. Investigators lacked information relating to urinary protein/albumin excretion as it could have helped us stage the study population using the KDIGO classification. Previous studies have demonstrated a positive link between the elevated baseline copeptin concentration and increased urinary albumin excretion in healthy individuals and among type 2 diabetic patients $[6,7]$. Similar results regarding the relationship between copeptin and albuminuria were found in another report from the same study group [8]. Therefore, without adjustment for albuminuria, it remains unknown if the association between the baseline copeptin and development of CKD is dependent or independent of albuminuria. Other important variables that could influence copeptin levels such as body mass index, serum and urine osmolality, serum sodium and cholesterol levels were not included in the models. Given the nature of the primary study population, they also had a smaller proportion of patients with diabetes and hypertension at baseline. Importantly, it is unclear whether adjustment for diabetes and hypertension developed during follow-up (time-dependent analysis or using marginal structural modeling) would have altered these associations.

Despite these limitations, this report points out the potential utility of copeptin measurement in identifying patients at risk for decline in kidney function. Although it is not a specific marker for disease, it could be used in conjunction with other screening methods to improve early detection and risk stratification of CKD if future studies could address the knowledge gap. Is copeptin a filtration marker or causally related to kidney function decline? Despite their attempt to adjust for GFR in the models to address this, the question remains open and cannot be answered by observational studies. However, some supportive evidence from experimental and human studies suggests potential causal relationship. In vasopressin gene-absent rats, infusion of V2-receptor agonist resulted in progression of CKD. Pharmacological blockage of V2 receptors improved urinary protein excretion, serum creatinine and structural damage of kidney caused by toxin injection in rats [9]. In human studies, the effectiveness of V2-receptor antagonist in slowing down ADPKD progression has been demonstrated in clinical trials. Further, AVP axis could be blocked with high water intake. Mechanistically, copeptin exert effects on urinary concentrating activity, renin-angiotensin system activation, glomerular hyperfiltration and tubular effects on sodium reabsorption, which in turn could impart detrimental effects on kidney function. Hence, lowering copeptin levels with water intake could provide beneficial effects for the kidneys.

Similar to the findings from observational studies, a recent pilot randomized study demonstrated that copeptin level was reduced in stage $3 \mathrm{CKD}$ patients by increasing water intake for 6 weeks. However, long-term data are warranted to see if the results of this pilot trial can be translated into an improvement of kidney function [10].

In summary, the report by Tasevska et al. [4] adds to the growing body of literature about the utility of copeptin as a marker to portend adverse outcomes. Further studies should confirm these findings along with addressing the limitations of this report. In addition, larger studies examining whether lowering copeptin in CKD with water intake could help us answer if the observed relationship between copeptin and decline in kidney function is causally related.

\section{Disclosure Statement}

S.D.N. is supported by a grant from the National Institutes of Health (NIDDK-R01DK101500). The content is solely the responsibility of the authors and does not necessarily represent the official views of the NIH. NIH did not have any role in study design; collection, analysis, and interpretation of data; writing the report; and the decision to submit the report for publication. The authors have no relevant financial interest in the contents of this study. 


\section{References}

1 Bankir L, Bouby N, Ritz E: Vasopressin: a novel target for the prevention and retardation of kidney disease? Nat Rev Nephrol 2013; 9:223-239.

2 Morgenthaler NG, Struck J, Jochberger S, Dünser MW: Copeptin: clinical use of a new biomarker. Trends Endocrinol Metab 2008; 19:43-49.

3 Fenske W, Wanner C, Allolio B, Drechsler C, Blouin K, Lilienthal J, Krane V: Copeptin levels associate with cardiovascular events in patients with ESRD and type 2 diabetes mellitus. J AM Soc Nephrol 2011;22:782790.

4 Tasevska I, Enhörning S, Persson M, Nilsson $\mathrm{PM}$, Melander O: Increased levels of copeptin, a surrogate marker of AVP, are associated with an increased risk of CKD in a healthy population. Am J Nephrol 2016; 44:22-28.
5 Roussel R, Matallah N, Bouby N, El Boustany R, Potier L, Fumeron F, Mohammedi K, Balkau B, Marre M, Bankir L, Velho G: Plasma copeptin and decline in renal function in a cohort from the community: the prospective D.E.S.I.R. study. Am J Nephrol 2015;42:107114.

6 Meijer E, Bakker SJ, Halbesma N, de Jong PE, Struck J, Gansevoort RT: Copeptin, a surrogate marker of vasopressin, is associated with microalbuminuria in a large population cohort. Kidney Int 2010;77:29-36.

7 Enhörning S, Bankir L, Bouby N, Struck J, Hedblad B, Persson M, Morgenthaler NG, Nilsson PM, Melander O: Copeptin, a marker of vasopressin, in abdominal obesity, diabetes and microalbuminuria: the prospective Malmö diet and cancer study cardiovascular cohort. Int J Obes (Lond) 2013;37:598-603.
8 Boertien WE, Riphagen IJ, Drion I, Alkhalaf A, Bakker SJ, Groenier KH, Struck J, de Jong PE, Bilo HJ, Kleefstra N, Gansevoort RT: Copeptin, a surrogate marker for arginine vasopressin, is associated with declining glomerular filtration in patients with diabetes mellitus (ZODIAC-33). Diabetologia 2013;56:16801688.

9 Okada T, Sakaguchi T, Hatamura I, Saji F, Negi S, Otani H, Muragaki Y, Kawachi H, Shigematsu T: Tolvaptan, a selective oral vasopressin V2 receptor antagonist, ameliorates podocyte injury in puromycin aminonucleoside nephrotic rats. Clin Exp Nephrol 2009;13:438-446.

10 Sontrop JM, Huang SH, Garg AX, Moist L, House AA, Gallo K, Clark WF: Effect of increased water intake on plasma copeptin in patients with chronic kidney disease: results from a pilot randomised controlled trial. BMJ Open 2015;5:e008634. 